\title{
Linear quadratic regulator and pole placement for stabilizing a cart inverted pendulum system
}

\author{
Mila Fauziyah', Zakiyah Amalia², Indrazno Siradjuddin ${ }^{3}$, Denda Dewatama ${ }^{4}$, Rendi Pambudi \\ Wicaksono $^{5}$, Erni Yudaningtyas ${ }^{6}$ \\ 1,2,3,4,5 Department of Electrical Engineering, Malang State Polytechnic of Malang, Indonesia \\ ${ }^{6}$ Department of Electrical Engineering, University of Brawijaya, Indonesia
}

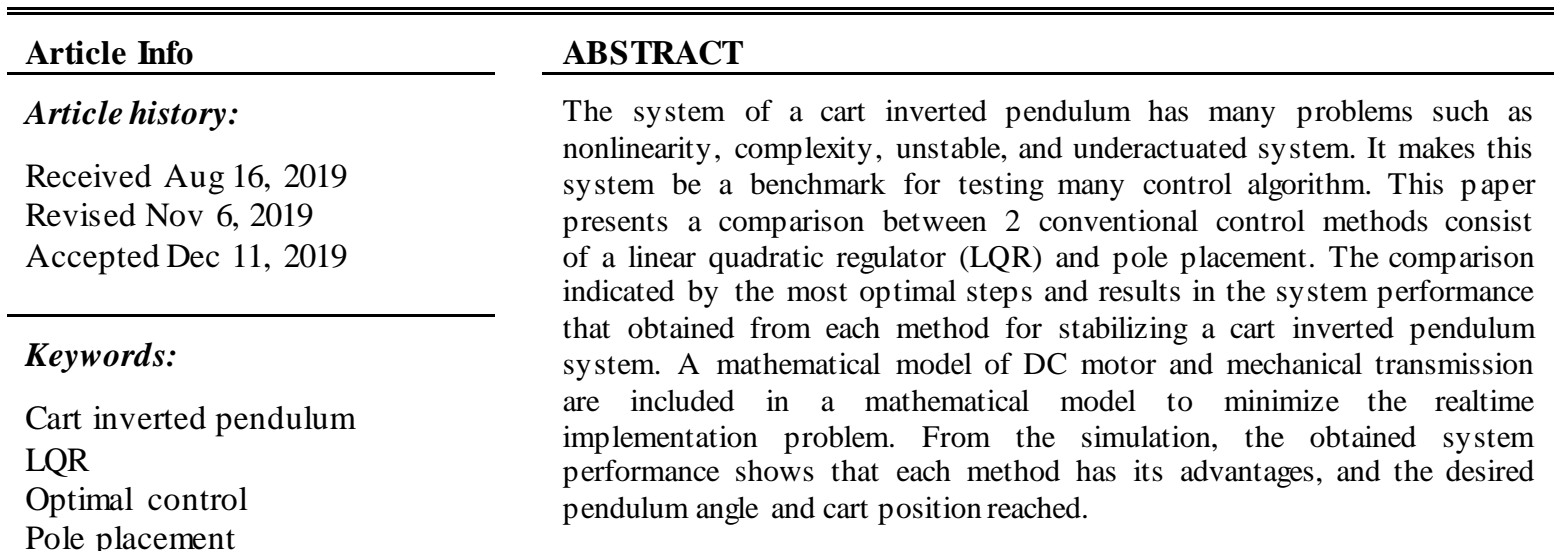

This is an open access article under the $\underline{C C B Y-S A}$ license.

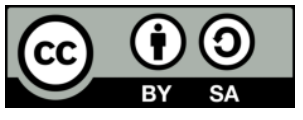

\section{Corresponding Author:}

Zakiyah Amalia,

Department of Electrical Engineering,

Politeknik Negeri Malang,

Soekarno Hatta 9, Malang, East Java 65141, Indonesia.

Email: zakiyah_amalia@polinema.ac.id

\section{INTRODUCTION}

Inverted Pendulum is a regular pendulum facing upwards, opposite the direction of gravity. This system is naturally unstable. One way to stabilize this system is to move the pivot point with a certain speed and acceleration in the direction of the vertical axis. The system of inverted pendulum is also nonlinear. By giving the same speed and acceleration to the system, the response from the pendulum angle of the rod varies. The system of an inverted pendulum is also underactuated because it has one input in the form of a vertical motion at the pivot point but has two outputs in the form of rod angles and the position of the pivot point. The nature of this inverted pendulum system makes the researchers make this system the choice of a test or benchmark tool for testing many control techniques since the 1973s to the present [1-2]. The PID control [3-6], fuzzy genetic algorithm [7-10], state-space control with pole placement methods [11-13], linear quadratic regulator (LQR) [14-16], linear quadratic gaussian (LQG) [17-20], observer [21-23], NCTL [24], flower pollination [25], and others have been used in this system.

This paper discusses the LQR and pole placement methods for stabilizing a cart inverted pendulum system. System modeling using the Lagrange equation. The equation of particle motion expressed by the Lagrange equation can be obtained by reviewing the kinetic energy and potential energy of the system without the need to review the forces acting on the system. After deriving the mathematical model using 
the Lagrange equation, the system of a cart inverted pendulum is brought to a state-space form for further control using the LQR and pole placement control methods.

\section{RESEARCH METHOD}

The system of a cart inverted pendulum is illustrated in Figure 1. There is a thrust $f$ which causes the cart to move on the horizontal axis $x$. This force is generated by a DC motor. $\omega_{1}, \omega_{2}$ is the rotational speed in the pulley transmission system, $\tau_{1}, \tau_{2}$ is the variable torque in the pulley transmission system, $l$ is the rod length, and $m$ is the pendulum mass centered on the rod end. This system has one input in the form of $f$ and two outputs, namely the pendulum angle $(\theta)$ and the cart position $(x)$.

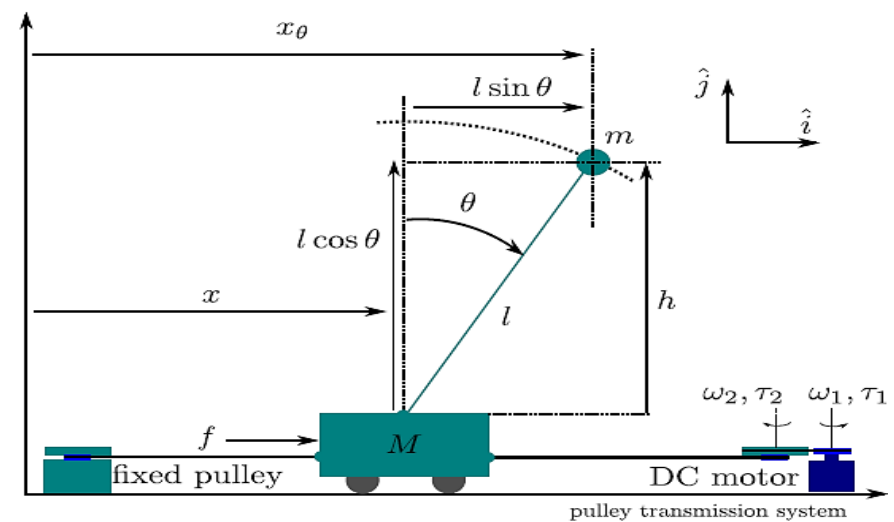

Figure 1. A cart inverted pendulum system

\subsection{Mathematical model}

\subsubsection{Lagrange equation on the system}

Mathematical modeling of an inverted pendulum system using the Lagrange equation. Lagrangian function $\mathcal{L}$ is expressed by kinetic energy $K$ and potential energy $P$ as follows:

$$
\mathcal{L}=K(\boldsymbol{q}, \dot{\boldsymbol{q}})-P(\boldsymbol{q})
$$

where $\boldsymbol{q}=\left\{\boldsymbol{q}_{1}, \cdots, \boldsymbol{q}_{\boldsymbol{i}}, \cdots, \boldsymbol{q}_{\boldsymbol{n}}\right\}$. The desired equation of motion is derived by using:

$$
\frac{d}{d t}\left(\frac{\partial \mathcal{L}}{\partial \dot{q}_{l}}\right)-\frac{\partial \mathcal{L}}{\partial q_{i}}=Q_{i}
$$

where $Q_{i}$ indicates the external force at the $q_{i}$ coordinate. The cart moves in the direction $i$ only, so the kinetic energy on the cart is expressed as:

$$
K_{M}=\frac{1}{2} M \dot{x}^{2}
$$

The pendulum moves in the direction $i$ and $j$, so that the kinetic energy in the pendulum is expressed as:

$$
K_{m}=\frac{1}{2} m\left(\dot{x}_{\theta}^{2}+\dot{h}^{2}\right)
$$

$x_{\theta}=x+l \sin \theta$ and $h=l \cos \theta$, then the equation becomes:

$$
K_{m}=\frac{1}{2} m\left(\dot{x}^{2}+2 \dot{x} l \dot{\theta} \cos \theta+l^{2} \dot{\theta}^{2}\right)
$$

So that the total kinetic energy of the inverted pendulum cart systemis :

$$
K=\frac{1}{2} M \dot{x}^{2}+\frac{1}{2} m\left(\dot{x}^{2}+2 \dot{x} l \dot{\theta} \cos \theta+l^{2} \dot{\theta}^{2}\right)
$$


The potential energy in the cart inverted pendulum system is only found in the pendulum mass $(m)$.

$$
\begin{aligned}
& P=m g h \\
& P=m g l \cos \theta
\end{aligned}
$$

Lagrange equation is obtained as follows:

$$
\mathcal{L}=\frac{1}{2} M \dot{x}^{2}+\frac{1}{2} m\left(\dot{x}^{2}+2 \dot{x} l \dot{\theta} \cos \theta+l^{2} \dot{\theta}^{2}\right)-m g l \cos \theta
$$

The cart inverted pendulum system has two generalised coordinates namely $x$ and $\theta$, so the Lagrange equation for each common coordinate is described as follows:

$$
\begin{aligned}
& \frac{d}{d t}\left(\frac{\partial \mathcal{L}}{\partial \dot{x}}\right)-\frac{\partial \mathcal{L}}{\partial x}=f \\
& \frac{d}{d t}\left(\frac{\partial \mathcal{L}}{\partial \dot{\theta}}\right)-\frac{\partial \mathcal{L}}{\partial \theta}=0
\end{aligned}
$$

After being derived, the general coordinates from the Lagrange equation of the system of a cart inverted pendulum can be rewritten to:

$$
\begin{aligned}
& f=(M+m) \ddot{x}+m l \ddot{\theta} \cos \theta-m l \dot{\theta}^{2} \sin \theta \\
& 0=m l(\ddot{x} \cos \theta-\dot{\theta} \dot{x} \sin \theta)+m l^{2} \ddot{\theta}-(-m \dot{x} l \dot{\theta} \sin \theta+m g l \sin \theta)
\end{aligned}
$$

Equations (11) and (12) are nonlinear equations because they have trigonometric terms sin () and cos (). The controller is designed to make $\theta$ as small as possible. With this as sumption, the linear Lagrange equation of the systemof a cart inverted pendulum becomes:

$$
\begin{aligned}
& f=(M+m) \ddot{x}+m l \ddot{\theta} \\
& 0=\ddot{x}+l \ddot{\theta}-g \theta
\end{aligned}
$$

\subsubsection{DC motor}

The armature DC motor equivalent circuit is shown in Figure 2. The open-loop system diagram in the form of state space is shown in Figure 3.

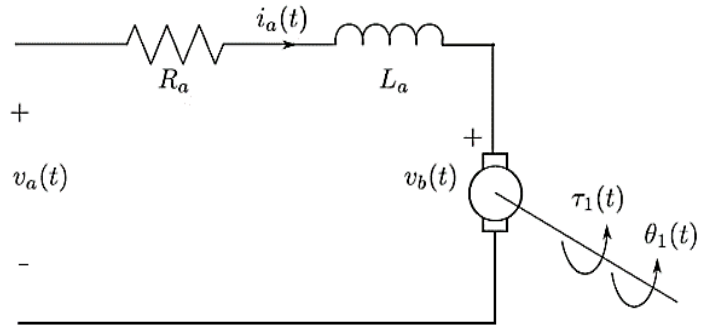

Figure 2. Armature DC motor

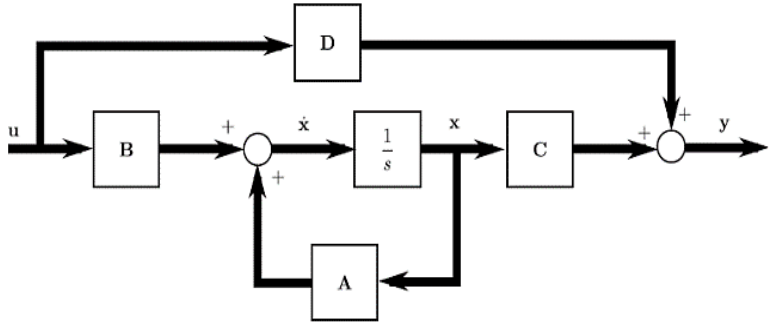

Figure 3. Open-loop system

The equation of the armarture DC motor circuit is

$$
i_{a} R_{a}+L_{a} \frac{d i_{a}}{d t}+v_{b}=v_{a}
$$

where $v_{b}=K_{b} \frac{d \theta_{1}}{d t}, \omega_{1}=\frac{d \theta_{1}}{d t}, \tau_{1}=K_{t} i_{a}$. So the equation of the amartur DC motor circuit becomes: 


$$
\frac{\tau_{1}}{K_{t}} R_{a}+L_{a} \frac{d^{2} \tau_{1}}{(d t)^{2}}+K_{b} \frac{d \theta_{1}}{d t}=v_{a}
$$

Generally, inductors in small rotors so that the armature circuit equation becomes:

$$
\tau_{1}=-K_{t} \frac{K_{b}}{R_{a}} \omega_{1}+\frac{K_{t}}{R_{a}} v_{a}
$$

The angular velocity of the motor $\omega_{1}$ is expressed in the speed of the cart $\dot{x}$ to be $\omega_{1}=\frac{\dot{x}}{r_{1}}$, and $K_{r}=\frac{K_{t}}{R_{a}}$ so the equation becomes:

$$
f=\frac{K_{r}}{r_{1}}\left(-K_{b} \frac{\dot{x}}{r_{1}}+v_{a}\right)
$$

So the Lagrange equation at the $x$ coordinate becomes:

$$
\frac{K_{r}}{r_{1}} v_{a}=(M+m) \ddot{x}+\frac{K_{r} K_{b}}{\left(r_{1}\right)^{2}} \dot{x}-m l \ddot{\theta}
$$

Simplified equations with $c_{1}=\frac{K_{r} K_{b}}{\left(r_{1}\right)^{2}}$ and $c_{2}=\frac{K_{r}}{r_{1}}$, becomes:

$$
\ddot{x}=\frac{1}{M}\left(c_{2} v_{a}-c_{1} \dot{x}-m g \theta\right)
$$

From (14), it can be obtained:

$$
\ddot{\theta}=\frac{1}{l}(g \theta-\ddot{x})
$$

Equation (20) is substituted to (21) so that it becomes:

$$
\begin{aligned}
& \ddot{\theta}=\frac{1}{l}\left(g \theta-\frac{1}{M}\left(c_{2} v_{a}-c_{1} \dot{x}-m g \theta\right)\right) \\
& \ddot{\theta}=-\frac{c_{2}}{M l} v_{a}-\frac{c_{1}}{M l} \dot{x}+\frac{(M+m) g}{M l} \theta
\end{aligned}
$$

The Lagrange equation for the system of a cart inverted pendulum has been obtained and subsequently converted into state space.

\subsubsection{State space representation}

The form of state space of a system is represented as:

$$
\begin{aligned}
& \dot{x}=A x+B u \\
& y=C x+D u
\end{aligned}
$$

where $\boldsymbol{x} \in R^{j}$ is a vector state, $\boldsymbol{u} \in R^{k}$ is a control input, $\boldsymbol{A} \in R^{j \times j}$ is a system matrix, $\boldsymbol{B} \in R^{j \times k}$ input matrix, $\boldsymbol{C} \in R^{p \times j}$ is an output matrix, and $\boldsymbol{y} \in R^{p}$ is an output vector. In a cart inverted pendulum system,

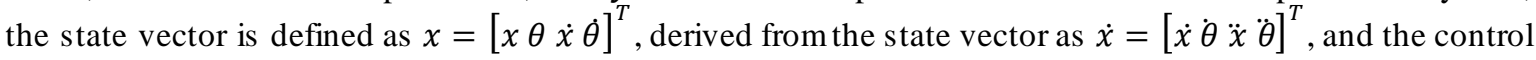
input $u=v_{a}$. From the Lagrange equation that has been obtained previously, the state space form of the systemof a cart inverted pendulum is;

$$
\boldsymbol{A}=\left[\begin{array}{cccc}
0 & 0 & 1 & 0 \\
0 & 0 & 0 & 1 \\
0 & -\frac{m g}{M} & -\frac{c_{1}}{M} & 0 \\
0 & \frac{(M+m) g}{M l} & \frac{c_{1}}{M l} & 0
\end{array}\right] \boldsymbol{B}=\left[\begin{array}{c}
0 \\
0 \\
\frac{c_{2}}{M} \\
-\frac{c_{2}}{M l}
\end{array}\right], \boldsymbol{C}=\left[\begin{array}{cccc}
1 & 0 & 0 & 0 \\
0 & 1 & 0 & 0
\end{array}\right] \boldsymbol{y}=[x \theta]^{T}, \boldsymbol{D}=\emptyset
$$




\subsection{Controller design}

\subsubsection{Linear quadratic regulator}

In the LQR method, the weight matrix $\boldsymbol{Q}$ and $R$ are determined to get the most optimal response. LQR is one method of designing a modern control system. The purpose of LQR is to regulate or make the output of y be zero with minimum input. In order to bring the system state from the initial state $x(t)=x(0)$ to the desired state $x(t)$ by minimizing $J$. In general, LQR problems are:

$$
J=\int_{0}^{\infty}\left[x^{T}(t) \boldsymbol{Q} x(t)+u^{T}(t) R u(t)\right]
$$

where $\boldsymbol{Q} \in R^{n \times n}$ and $R \in R^{n \times m}$. $Q$ and $R$ each determine the performance and energy control needed. The optimal gain of feedback $K$ can be obtained by;

$$
\boldsymbol{K}=R^{-1} \boldsymbol{B}^{T} \boldsymbol{P}
$$

where $P$ is a positive definite matrix which is a solution of the Riccati equation with:

$$
\boldsymbol{A}^{T} \boldsymbol{P}+\boldsymbol{P A}-\boldsymbol{P B} R^{-1} \boldsymbol{B}^{T} \boldsymbol{P}+\boldsymbol{Q}=0
$$

\subsubsection{Pole placement}

Pole placement is a control method that is also used to get the most optimal response. Pole placement is also a modern control method. The location of the pole can be used as a reference to find out whether a system is stable or not. A stable system has a pole to the left of the imaginary axis, while a system that has a pole to the right of the imaginary axis is an unstable system. Therefore, there needs to be a control method to bring the pole which is to the right of the imaginary axis to the left of the imaginary axis so that the system becomes stable. Pole placement is a control method that can bring the pole to the right position. By using the gain feedback $\boldsymbol{K}$, the desired design criteria of the system can be met. The polynomial characteristics can be described as:

$$
|s \boldsymbol{I}-\boldsymbol{A}|=s^{n}+a_{1} s^{n-1}+\cdots+a_{n-1} s+a_{n}
$$

where $\boldsymbol{I}$ is the identity matrix and a is the polynomial coefficient. If the desired eigenvalues $\left[\mu_{1}, \cdots, \mu_{n}\right]$ can be considered, then the desired characteristics of the polynomial are;

$$
\prod_{i=1}^{n}\left(s-\mu_{1}\right)=s^{n}+\propto_{1} s^{n-1}+\cdots+\propto_{n-1} s+\propto_{n}
$$

The feedback matrix gain required by $\boldsymbol{K}$ can be calculated using;

$$
\boldsymbol{K}=\left[\propto_{n}-a_{n}\left|\propto_{n-1}-a_{n-1}\right| \cdots\left|\propto_{2}-a_{2}\right| \propto_{1}-a_{1}\right] \boldsymbol{T}^{-1}
$$

The LQR method and pole placement use the feedback matrix. The feedback matrix in question can be described as the matrix $\boldsymbol{K}$ in Figure 4. To support the performance of the system, the pre-gain $N$ matrix is also shown in Figure 5.

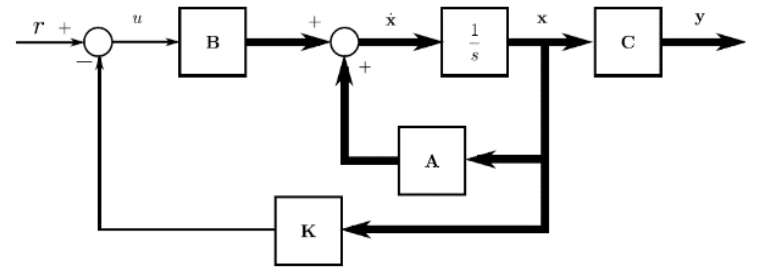

Figure 4. Closed-loop system

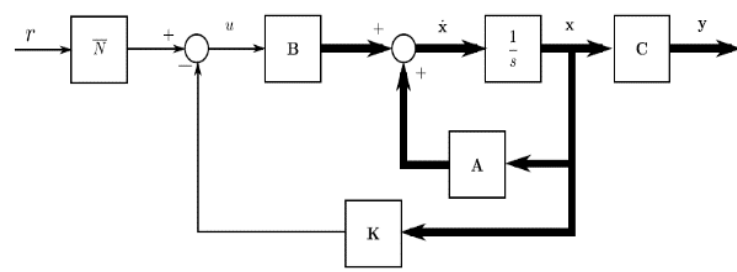

Figure 5. A closed-loop system with pregain

Controllability and observability of the system is something that must be met before the control is done. The controllability of the system is indicated by rank $(\boldsymbol{C})=n$, where $n$ is equal to the number 
of state variables for all initial state values. The rank of observability ( $\boldsymbol{O}$ ) must also be the same as the number of states,

$$
\begin{aligned}
& \mathcal{C}=\left[\boldsymbol{B}|\boldsymbol{A B}| \boldsymbol{A}^{2} \boldsymbol{B}|\cdots| \boldsymbol{A}^{n-1} \boldsymbol{B}\right] \\
& \mathcal{O}=\left[\boldsymbol{C}|\boldsymbol{C A}| \boldsymbol{C} \boldsymbol{A}^{2}|\cdots| \boldsymbol{C} A^{n-1}\right]
\end{aligned}
$$

\section{RESULT AND ANALYSIS}

The parameters of the system of a cart inverted pendulum are determined for simulations in the Linear Quadratic Regulator and the pole placement methods as in Table 1.

Table 1. Cart inverted pendulum parameters

\begin{tabular}{cc}
\hline Parameters & Value \\
\hline$R_{a}$ & $1.25 \Omega$ \\
$r_{1}$ & $0.02 \mathrm{~m}$ \\
$K_{b}$ & $0.015 \mathrm{~V} . \mathrm{s} / \mathrm{rad}$ \\
$M$ & $1.2 \mathrm{Kg}$ \\
$K_{t}$ & $0.015 \mathrm{~N} . \mathrm{m} / \mathrm{A}$ \\
$m$ & $0.2 \mathrm{Kg}$ \\
$l$ & $0.3 \mathrm{~m}$ \\
\hline
\end{tabular}

The controllability and observability of the system of a cart inverted pendulum is obtained namely $\operatorname{rank}(\boldsymbol{C})=\operatorname{rank}(\boldsymbol{O})=4$ so that control can be done with;

$$
\mathcal{C}=\left[\begin{array}{cccc}
0 & 0.5 & -0.1875 & 2.795 \\
0 & -1.667 & 0.625 & -63.81 \\
0.5 & -0.187 & 2.795 & -2.07 \\
-1.667 & 0.625 & -63.81 & 27.34
\end{array}\right]
$$$$
\boldsymbol{O}=\left[\begin{array}{cccc}
1 & 0 & 0 & 0 \\
0 & 1 & 0 & 0 \\
0 & 0 & 1 & 0 \\
0 & 0 & 0 & 1 \\
0 & -1.635 & -0.375 & 0 \\
0 & 38.15 & 1.25 & 0 \\
0 & 0.6131 & 0.1406 & -1.635 \\
0 & -2.0438 & -0.4688 & 38.15
\end{array}\right]
$$

It is known that one of the poles of the system is to the right of the imaginary axis. The pole is worth $(0.6 .1512,-6.2049,-0.3213)$ so control is needed. Control is done with the desired output in the form of a pendulum angle at the balance point and the position of the cart is at 0.3 meters. The pole-zero mapping from the systemof a cart inverted pendulum is shown in Figure 6.

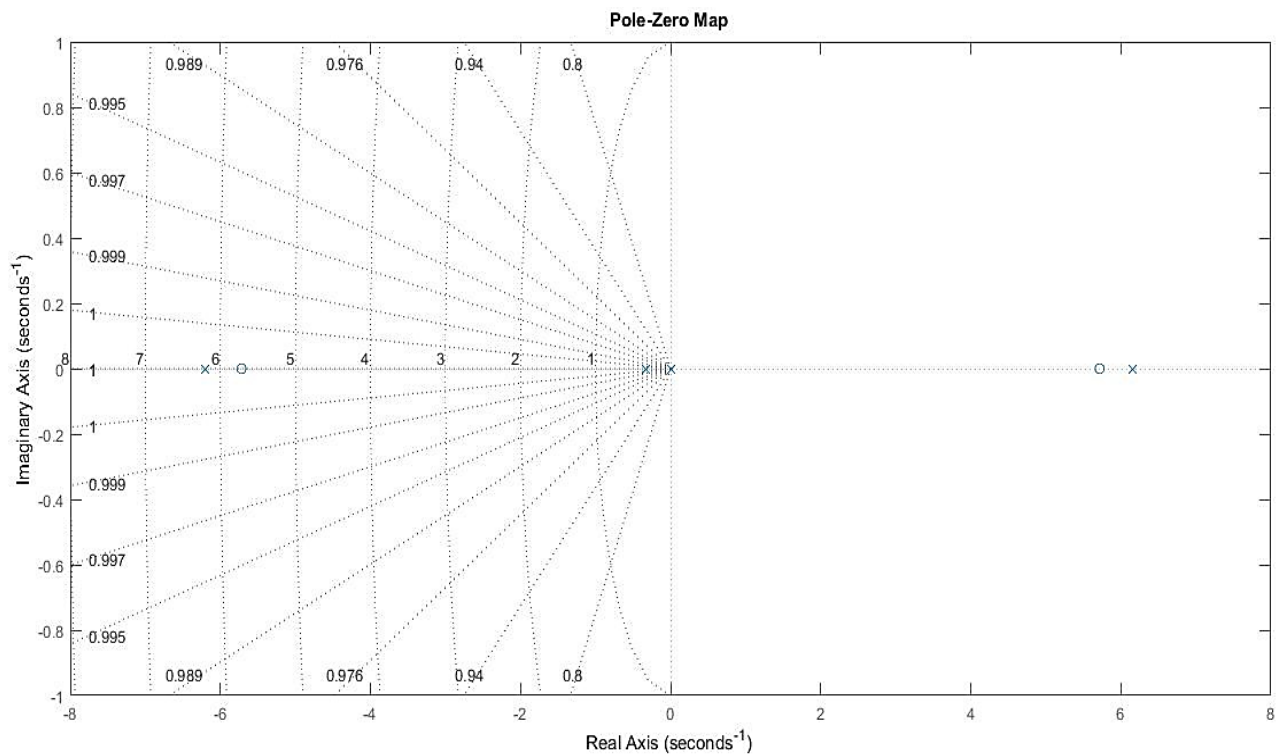

Figure 6. Pole-zero mapping 


\subsection{Simulation of the $L Q R$ method}

The simulation using the LQR method uses 5 variations of the weight matrix $\boldsymbol{Q}$ and $R$ as in Table 2 . The weighting matrix $\boldsymbol{Q}=\left[q_{1}, q_{2}, q_{3}, q_{4}\right]$ that is changed is $q_{1}, q_{2}$ because that affects state $x, \theta$ directly. The combination produces a feedback matrix $(\boldsymbol{K})$ and pre-gain $(N)$ as Table 3 . From the 5 configurations in Table 2 and Table 3, the systemresponse was obtained as shown in Figures 7, 8, and 9.

Table 2. LQR parameter configuration

\begin{tabular}{cccc}
\hline No & $q_{1}$ & $q_{2}$ & $R$ \\
\hline 1 & 0.1 & 1 & 1 \\
2 & 1 & 1 & 1 \\
3 & 1 & 2 & 1 \\
4 & 1 & 2 & 10 \\
5 & 2 & 2 & 10 \\
\hline
\end{tabular}

Table 3. $N, \boldsymbol{K}$ parameters

\begin{tabular}{ccrccc}
\hline No & $N$ & $K 1$ & $K 2$ & $K 3$ & $K 4$ \\
\hline 1 & -0.3162 & -0.3162 & -50.5224 & -2.2810 & -8.2416 \\
2 & -1.0000 & -1.0000 & -53.6364 & -3.3636 & -8.7875 \\
3 & -1.0000 & -1.0000 & -53.6561 & -3.3644 & -8.7892 \\
4 & -0.3162 & -0.3162 & -50.5062 & -2.2807 & -8.2402 \\
5 & -0.4472 & -0.4472 & -51.2154 & -2.5234 & -8.3645 \\
\hline
\end{tabular}

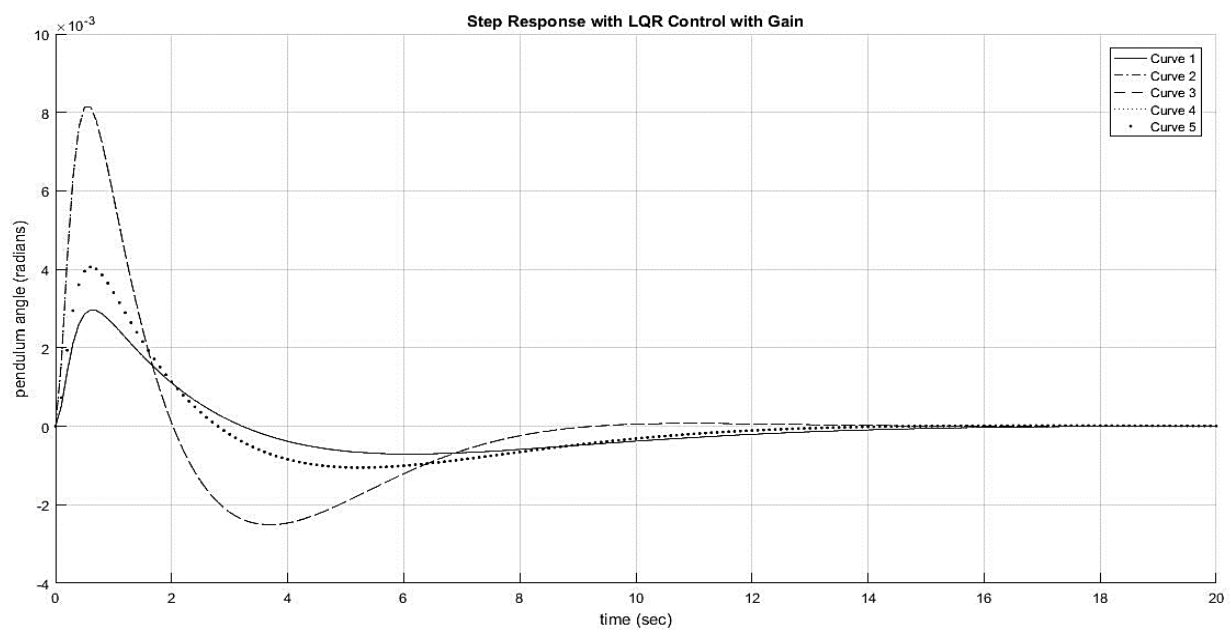

Figure 7. The angle response of the pendulum with the LQR method

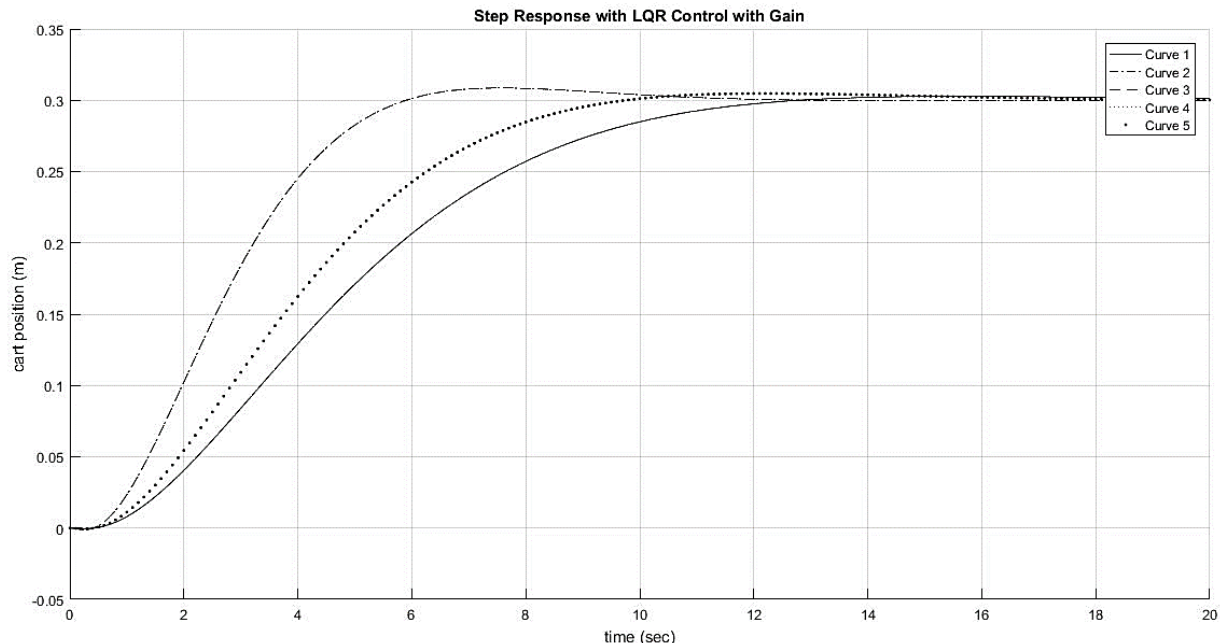

Figure 8. Cart position response with the LQR method 


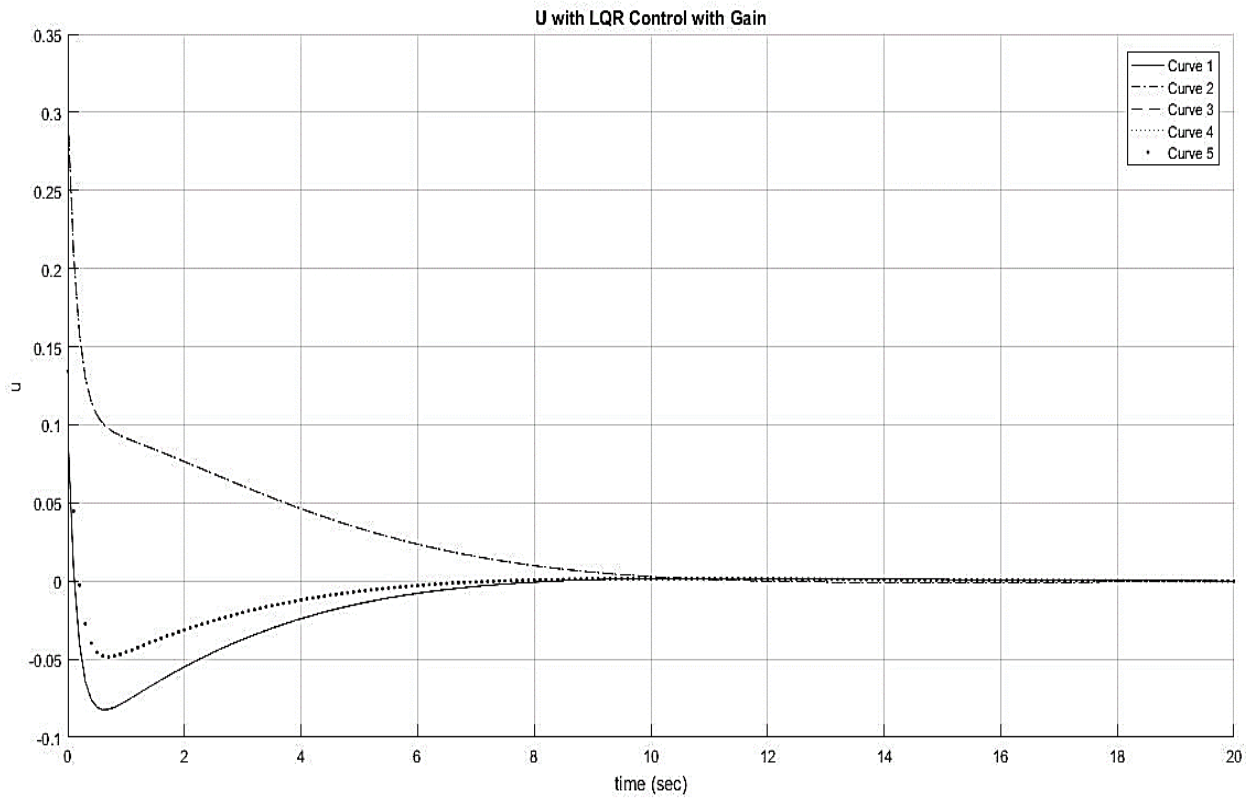

Figure 9. Control signal with the LQR method

From Figure 7, 8, and 9 it can be seen that the greater the $\boldsymbol{Q}$ and $R$ weighting matrix, the faster the system response to reach the desired state and the greater the control signal. However, by choosing a small $R$, the control signal can be minimized. Therefore, choosing the right $\boldsymbol{Q}$ and $R$ matrix will produce an optimal controller.

\subsection{Pole placement method simulation}

Simulation using the pole placement method uses 5 variations of the pole matrix $S_{1}, s_{2}, s_{3}, s_{4}$ as in Table 4. Poles are chosen randomly with negative values. The combination produces a feedback matrix $(\boldsymbol{K})$ and pre-gain $(N)$ as in Table 5 .

Table 4. Pole placement parameters

\begin{tabular}{ccccc}
\hline No & $S_{1}$ & $S_{2}$ & $S_{3}$ & $S_{4}$ \\
\hline 1 & $-6.18+0.19 \mathrm{i}$ & $-6.18-0.19 \mathrm{i}$ & $-0.48+0.43 \mathrm{i}$ & $-0.48-0.43 \mathrm{i}$ \\
2 & $-6.18+0.19 \mathrm{i}$ & $-6.18-0.19 \mathrm{i}$ & $-0.48+0.43 \mathrm{i}$ & -0.489 \\
3 & $-6.18+0.19 \mathrm{i}$ & $-6.18-0.19 \mathrm{i}$ & $-0.48+0.43 \mathrm{i}$ & -0.189 \\
4 & $-6.18+0.19 \mathrm{i}$ & $-6.18-0.19 \mathrm{i}$ & $-0.48+0.43 \mathrm{i}$ & -0.089 \\
5 & $-6.18+0.19 \mathrm{i}$ & $-6.18-0.19 \mathrm{i}$ & -0.48 & -0.089 \\
\hline
\end{tabular}

Table 5. $N, \boldsymbol{K}$ parameters

\begin{tabular}{cccccc}
\hline No & $N$ & $K 1$ & $K 2$ & $K 3$ & $K 4$ \\
\hline 1 & -1.0000 & -1.0000 & -53.65 & -3.3644 & -8.7891 \\
2 & $-0.56+0.49 \mathrm{i}$ & $-0.56+0.49 \mathrm{i}$ & $-53.41+3.48 \mathrm{i}$ & $-3.22+1.17 \mathrm{i}$ & $-8.74+0.61 \mathrm{i}$ \\
3 & $-0.21+0.19 \mathrm{i}$ & $-0.24+0.19 \mathrm{i}$ & $-50.99+3.32 \mathrm{i}$ & $-2.41+1.07 \mathrm{i}$ & $-8.32+0.58 \mathrm{i}$ \\
4 & $-0.10+0.09 \mathrm{i}$ & $-0.10+0.09 \mathrm{i}$ & $-50.18+3.26 \mathrm{i}$ & $-2.13+1.04 \mathrm{i}$ & $-8.18+0.57 \mathrm{i}$ \\
5 & -0.1029 & -0.1029 & -50.1898 & -2.1388 & -8.1815 \\
\hline
\end{tabular}

From the 5 configurations in Table 4 and Table 5, the system response is obtained as shown in Figures 10, 11 and 12. From Figures 10, 11, and 12 it can be seen that the increasingly negative pole position produces an increasingly rapid response at the angle of the pendulum, but the overshoot also gets bigger. By looking at the control signal from each pole, the optimal controller can be selected. 


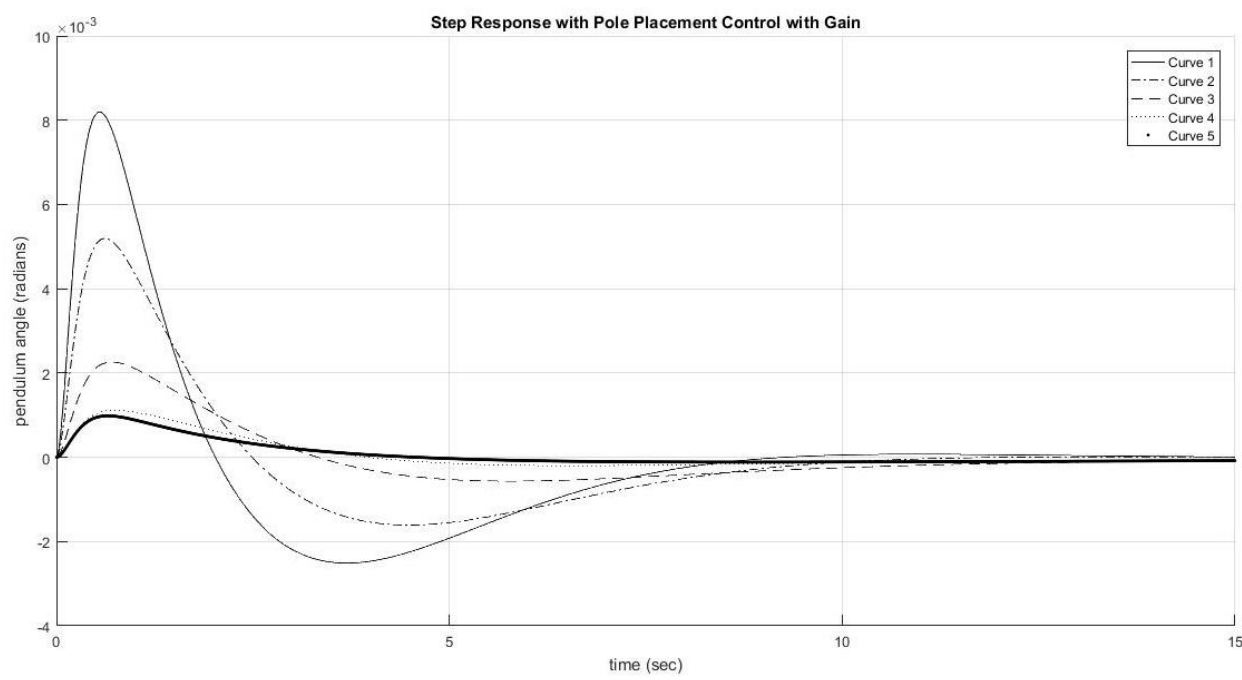

Figure 10. The angle response of the pendulum using the pole placement method

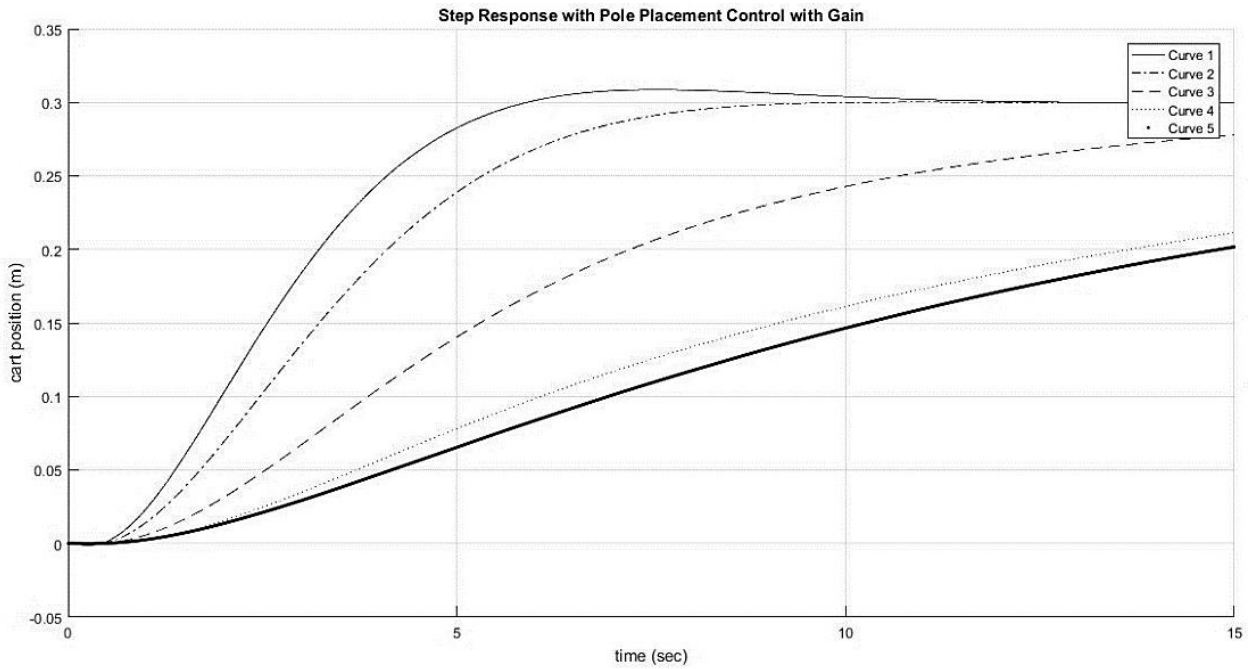

Figure 11. Response cart with the pole placement method

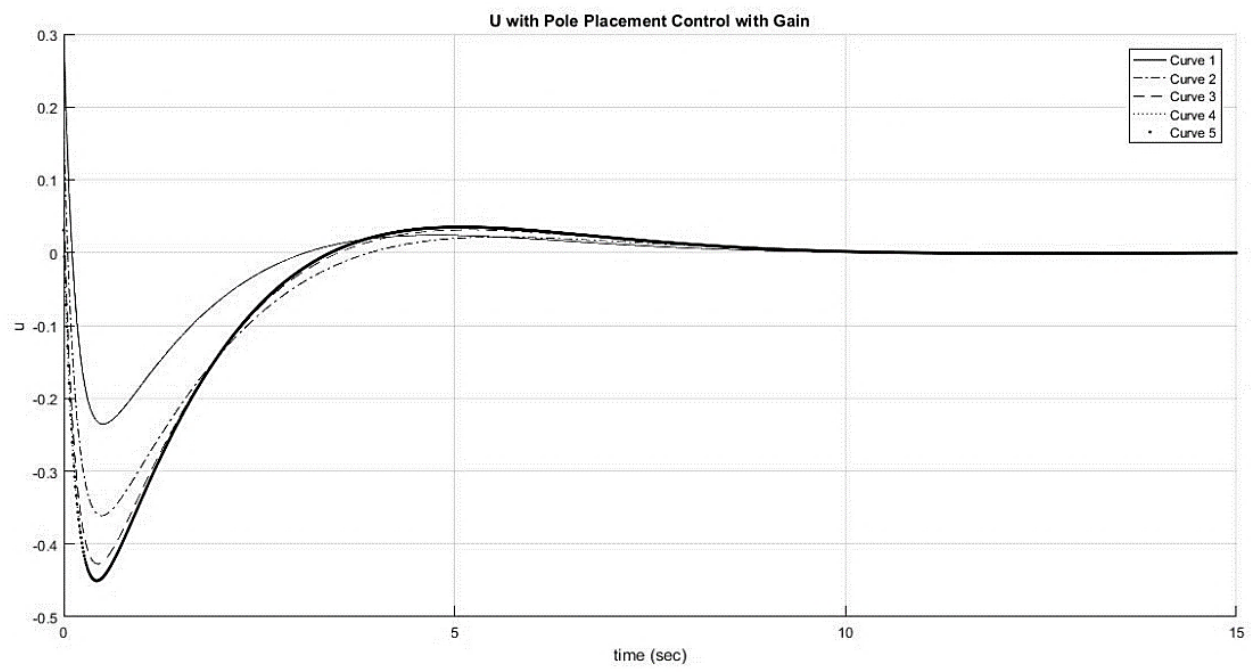

Figure 12. Control signal with the pole placement method 


\section{CONCLUSION}

Linear quadratic regulator and pole placement for stabilizing a cart inverted pendulum system has been successfully conducted. By using LQR, the system response can be obtained by selecting the weight matrix $\mathrm{Q}$ and $\mathrm{R}$ which is then continued with the calculation to get the desired pole. With the pole placement method, the system response can be chosen by directly selecting the desired pole, although it is necessary to repeatedly determine the pole to get the most optimal results. Both methods can produce optimal system response with fast systemresponse and minimal control signals.

\section{REFERENCES}

[1] H. Hemami, F. Weimer and S. Koozekanani, "Some aspects of the inverted pendulum problem for modeling of locomotion systems," IEEE Transactions on Automatic Control. December, vol. 18, no. 6, pp. 658-661, 1973.

[2] D. Du et al., "Real-Time Hळ control of networked inverted pendulum visual servo systems," IEEE Transactions on Cybernetics, pp. 1-14, 2019.

[3] I. Siradjuddin et al., "Stabilising a cart inverted pendulum with an augmented PID control scheme," MATEC Web of Conferences. EDP Sciences, vol. 197, pp. 1-7,2018.

[4] J. J. Wang, "Simulation studies of inverted pendulum based on PID controllers," Simulation Modelling Practice and Theory, vol. 19, no. 1, pp. 440-449, 2011.

[5] M.El-Bardini and A. M.El-Nagar, "Interval type-2 fuzzy PID controller for uncertain nonlinear inverted pendulum system,” ISA Transactions, vol. 53, no. 3, pp. 732-743, 2014.

[6] L. B. Prasad, B. Tyagi, and H. O. Gupta, "Optimal control of nonlinear inverted pendulum system using PID controller and LQR: performance analysis without and with disturbance input," Int. J. of Automation and Computing, vol. 11, no. 6, pp.661-670, 2014.

[7] L. H. Yu and F. Jian, "An inverted pendulum fuzzy controller design and simulation," International Symposium on Computer, Consumer and Control, pp. 557-559, 2014.

[8] M. Muhammad et al., "Velocity control of a two-wheeled inverted pendulum mobile robot: A fuzzy model-based approach," Bulletin of Electrical Engineering and Informatics, vol. 8, no. 3, pp. 808-817, 2019.

[9] R. Fierro et al., "Optimization of membership functions for the fuzzy controllers of the water tank and inverted pendulum with differents PSO variants," TELKOMNIKA Telecommunication Computing Electronics and Control, vol. 11 , no. 4, pp. 699-714, 2013.

[10] H. Li, M. Zhihong, and W. Jiayin, "Variable universe adaptive fuzzy control on the quadruple inverted pendulum," Science in China Series E: Technological Sciences, vol. 45, no. 2, pp. 213-224, 2002.

[11] M. Shehu et al., "LQR, double-PID and pole placement stabilization and tracking control of single link inverted pendulum," IEEE International Conference on Control System, Computing and Engineering, pp. 218-223, 2015.

[12] I. Siradjuddin et al., "Stabilising a cart inverted pendulum system using pole placement control method," Int. Conf. on Quality in Research (QiR): Int. Symposium on Electrical and Computer Enginee., pp. 197-203, 2017.

[13] M. W. Spong, P. Corke, and R. Lozano., "Nonlinear control of the reaction wheel pendulum," Automatica, vol. 37 , no. 11 pp. 1845-1851, 2001.

[14] E. V. Kumar, E. Vinodh, and J. Jerome, "Robust LQR controller design for stabilizing and trajectory tracking of inverted pendulum," Procedia Engineering, vol. 64, pp. 169-178, 2013.

[15] I. Siradjuddin et al., "State space control using lqr method for a cart-inverted pendulum linearised model," International Journal of Mechanical and Mechatronics Engineering, vol. 17, no. 1, pp. 119-126, 2017.

[16] H. Wang et al., "Design and simulation of LQR Controller with the Linear Inverted Pendulum," International Conference on Electrical and Control Engineering, pp.699-702, 2010.

[17] A. Odry, E. Burkus, and P. Odry, "LQG control of a two-wheeled mobile pendulum system," The Fourth International Conference on Intelligent Sy stems and Applications, pp. 105-112, 2015.

[18] C. Mahapatra, S. Chauhan and B. Hemakumar, "Servo control and stabilization of linear inverted pendulum on a cart using LQG," Int. Conference on Power Energy, Environment and Intelligent Control, pp. 783-788, 2018.

[19] R. Eide et al., "LQG control design for balancing an inverted pendulum mobile robot," Intelligent Control and Automation, vol. 2, no. 2, pp. 160-166, 2011.

[20] Sazonov, E. S., P. Klinkhachorn, and R. L. Klein, "Hybrid LQG-neural controller for inverted pendulum system," Proceedings of the 35th Southeastern Symposium on System Theory, pp. 206-210, 2003.

[21] I. Siradjuddin et al., "State-feedback control with a full-state estimator for a cart-inverted pendulum system," International Journal of Engineering \& Technology, vol. 7, no. 4.44, pp. 203-209, 2018.

[22] J. Huang et al., "Nonlinear Disturbance observer-based dynamic surface control of mobile wheeled inverted pendulum," IEEE Transactions on Control Systems Technology, vol. 23, no. 6, pp. 2400-2407, 2015.

[23] W. Maletinsky, M. F. Senning, and F. Wiederkehr, "Observer based control of a double pendulum," IFAC Proceedings, vol. 14, no. 2, pp.3383-3387, 1981.

[24] N. H. Jalani, "NCTF-FL controller for pendulum balancing system," Bulletin of Electrical Engineering and Informatics, vol. 4, no. 4, pp.355-362, 2015.

[25] S. Kavirayani, and G. V. Kumar., "Flower pollination for rotary inverted pendulum stabilization with delay," TELKOMNIKA Telecommunication Computing Electronics and Control, vol. 15, no. 1, 2017. 